\title{
PERFIL HEPÁTICO E ELETROLÍTICO DE CÃES IDOSOS SADIOS OU COM DOENÇA RENAL CRÔNICA TRATADOS COM O ANTIOXIDANTE $\mathrm{N}$-ACETILCISTEÍNA
}

\author{
André Luiz Baptista Galvão', Marileda Bonafim Carvalho
}

\author{
1 UNESP - Jaboticabal \\ Correspondência: André Luiz Baptista Galvão: andrelgalvao@hotmail.com
}

\begin{abstract}
RESUMO: A N-acetilcisteína (NAC) tem sido empregada na proteção renal e hepática em modelos experimentais. O objetivo do presente trabalho foi avaliar os efeitos do tratamento com NAC sobre o perfil hepático e eletrolítico de cães idosos saudáveis ou com doença renal crônica (DRC) naturalmente adquirida, clinicamente estáveis. Quatro grupos de cães idosos (9 a 15 anos) foram avaliados, compreendendo o grupo normal controle $(\mathrm{N}-\mathrm{C} ; \mathrm{n}=4)$, grupo normal tratado $(\mathrm{N}-\mathrm{T} ; \mathrm{n}=5)$, grupo doente renal crônico controle (DRC-C; $n=5)$ e grupo doente renal crônico tratado (DRC-T; $n=$ 4). Os grupos N-T e DRC-T foram submetidos ao tratamento com NAC (VO) na dose de $10 \mathrm{mg} / \mathrm{kg}$ b.i.d, durante 60 dias. Os grupos N-C e DRC-C não foram submetidos a qualquer tipo de tratamento. A concentração sérica de proteína total (Spt), albumina (SAlb), alanina aminotransferase (SALT), fosfatase alcalina (SFA), sódio (SNa), potássio (SK), fósforo (SP), cálcio total (SCat) e cálcio iônico (SCai) foram os parâmetros avaliados previamente, 15, 30, 45 e 60 dias após o tratamento com NAC. Os dados foram comparados estatisticamente por ANOVA para medidas repetidas, seguido pelo teste Tukey $(\alpha=0,05)$. A NAC não exerceu qualquer efeito sobre o perfil hepático dos cães estudados. A SNa no grupo N-T (149 $\pm 4,99 \mathrm{mg} / \mathrm{dL})$ foi superior, em relação ao $\mathrm{N}-\mathrm{C}(141 \pm 1,32$ $\mathrm{mg} / \mathrm{dL})(\mathrm{P}<0,05)$. Na comparação múltiplas de médias do SK e SCat no grupo $\mathrm{N}-\mathrm{T}$ a média aos 15 dias de tratamento $(4,94 \pm 0,38 \mathrm{mEq} / \mathrm{L} ; 12,37 \pm 0,45 \mathrm{mEq} / \mathrm{L})$ foi superior em relação a média basal $(4,26 \pm 0,22 \mathrm{mEq} / \mathrm{L} ; 10,13 \pm 0,56 \mathrm{mEq} / \mathrm{L})$. Concluiu-se que a NAC, em relação aos parâmetros analisados, promoveu em cães idosos sadios, o aumento de SK e SCat.
\end{abstract}

Palavras-chave: albumina; cálcio; potássio; sódio

\section{HEPATIC AND ELECTROLYTE PROFILE OF OLDER DOGS HEALTHY OR WITH CHRONIC KIDNEY DISEASE TREATED WITH ANTIOXIDANT N- ACETILCYSTEINE}

\begin{abstract}
N}$-acetylcysteine (NAC) has been used in hepatic and renal protection in experimental models. The objective of this study was to evaluate the effects of NAC treatment on profile hepatic and electrolytic for older dogs healthy or with chronic kidney disease (CKD) naturally acquired and clinically stable. Four groups of aged dogs ( $9-15$ years) were evaluated, including the control normal group $(\mathrm{N}-\mathrm{C}, \mathrm{n}=4)$, normal group treated $(\mathrm{N}-\mathrm{T}, \mathrm{n}=5)$, group control chronic kidney disease (CKD-C, $\mathrm{n}=5$ ) and the group treated chronic kidney disease (CKD-T, $n=4)$. The groups N-T and CKD-T were submitted to treatment with NAC (VO) at the dose of $10 \mathrm{mg} / \mathrm{kg}$ bid for 60 days. The groups N-C and CKD-C were not submitted to any type of treatment. The serum concentration total protein (SPt), albumin (SAlb), alanina amino-transferase (SALT), alkaline phosphatase (SFA), sodium (SNa), potassium (SK), phosphorus (SP), total calcium (SCat) and the calcium ionc (SCai) were previously evaluated, 15, 30, 45 and 60 days after being treated with NAC. The data were compared by ANOVA for repeated measures followed by the Tukey test $(\alpha=0,05)$. The NAC had no effect on the hepatic profile of the dogs studied. The SNa in the N-T $(149 \pm 4,99 \mathrm{mg} / \mathrm{dL})$ was higher compared to N-C (141 $\pm 1,32 \mathrm{mg} / \mathrm{dL})(\mathrm{P}<0,05)$. In multiple comparisons of mean SK and SCat in the $\mathrm{N}-\mathrm{T}$ average at 15 days of treatment $(4,94 \pm 0,38 \mathrm{mEq} / \mathrm{L} ; 12,37 \pm 0,45 \mathrm{mEq} / \mathrm{L})$ was higher than the average baseline $(4,26 \pm$ $0,22 \mathrm{mEq} / \mathrm{L} ; 10,13 \pm 0,56 \mathrm{mEq} / \mathrm{L})$. It was conclude that the antioxidant NAC promoted in older dogs healthy, increase of SK and SCat.
\end{abstract}

Key Words: albumin; calcium; potassium; sodium 


\section{INTRODUÇÃO}

A N-acetilcisteína (NAC) é um antioxidante que tem sido empregado na proteção renal em modelos experimentais de insuficiência renal aguda isquêmica, em estudos da lesão renal aguda tóxica por contraste radiográfico em animais e humanos (Vattimo et al., 2004). Os rins de cães com doença renal crônica (DRC) estão sujeitos a lesões adicionais provocadas por transtornos vasculares que contribuem para a progressão da doença (Newman, 2012). Na DRC, a partir do estágio 2, fazem parte da síndrome urêmica alguns sinais de desequilíbrio eletrolítico e de comprometimento hepático dentre outros (Newman, 2012). No perfil hepático, a NAC, também tem demonstrado resultados promissores, como protetor de lesão por isquemia e reperfusão (Didoné et al., 2002; Zeng et al., 2009). Este antioxidante é um aminoácido, tiol, agente mucolítico e precursor da L-cisteína e glutationa reduzida nas células. Trata-se de uma fonte de grupos sulfidril removedores de espécies reativas do metabolismo do oxigênio (ERMO) (Locatelli et al., 2003).

Estudar 0 efeito deste antioxidante sobre o perfil hepático e eletrolítico de cães idosos sadios ou DRC, naturalmente adquirida, e clinicamente estáveis, foi objetivo do presente trabalho.

\section{MATERIAL E MÉTODOS}

Foram avaliados 18 cães idosos ( 9 a 15 anos) sadios ou com DRC naturalmente adquirida, sem infecção urinária ou outra doença concorrente, e com quadro clínico estável.

Os animais foram divididos em quatro grupos, o normal controle (N-C; $n$ = 4), normal tratado (N-T;n=5), DRC controle (DRC-C; $n=5$ ) e DRC tratado
(DRC-T; $n=4)$. A concentração sérica de creatinina (Scr) e ureia (Sureia) na avaliação basal foi o parâmetro utilizado para a distribuição dos animais nos grupos, conforme critérios de classificação para cães com DRC estabelecidos pela Iris (2009).

Desse modo, a média \pm erro padrão da Scr e Sureia dos grupos N-T e DRC-T foram $1,03 \pm 0,08 \mathrm{mg} / \mathrm{dL}$ e $21,98 \pm 5,53 \mathrm{mg} / \mathrm{dL}, 2,03 \pm 0,49 \mathrm{mg} / \mathrm{dL}$ e $65,39 \pm 21,29 \mathrm{mg} / \mathrm{dL}$, respectivamente. Para os grupos N-C e DRC-C a média \pm erro padrão da Scr e Sureia foram de $0,76 \pm 0,10 \mathrm{mg} / \mathrm{dL}$ e $18,01 \pm 2,12$ $\mathrm{mg} / \mathrm{dL}, 2,46 \pm 0,40 \mathrm{mg} / \mathrm{dL}$ e $88,90 \pm$ $13,39 \mathrm{mg} / \mathrm{dL}$, respectivamente.

Os grupos N-T e DRC-T receberam como tratamento único a NAC na dose de $10 \mathrm{mg} / \mathrm{kg}$, V.O., b.i.d, durante 60 dias. Os grupos N-C e DRC$C$ não receberam qualquer tipo de tratamento. $\mathrm{O}$ tratamento experimental foi feito com 0 antioxidante $\mathrm{N}$ acetilcisteína (Fluimucilß) suspensão 40 $\mathrm{mg} / \mathrm{mL}$ - Laboratório Zambon). A dosagem foi estabelecida de acordo com o descrito por Papich (2007). A concentração sérica de proteína total (SPt), albumina (SAlb), alanina aminotransferase (SALT), fosfatase alcalina (SFA), fósforo (SP), cálcio total (SCat) e cálcio iônico (SCai) foram os parâmetros avaliados previamente (basal), 15, 30, 45 e 60 dias após 0 tratamento com NAC.

A condução do experimento seguiu um delineamento em blocos casualizados e, para cada animal, teve duração de 61 dias.

As amostras de soro foram processadas para determinação de creatinina (método Jaffé modificado), ureia (método enzimático), proteína total (método biureto), albumina (método verde de bromocresol), alanina aminotransferase (método Reitman e Frankel), fosfatase alcalina (método Roy modificado), cálcio total (método da 
cresolftaleina complexona), e fósforo (método do fosfomolibdato). Todas as análises bioquímicas foram feitas com os conjuntos de reagentes do o sistema Labtest (Labtest Diagnóstica S.A., Lagoa Santa - MG) para diagnóstico. Para as leituras empregou-se espectrofotômetro (Labtest Diagnóstica S.A., Lagoa Santa - MG) semiautomático.

As concentrações séricas de sódio, potássio e cálcio iônico foram feitas pelo método de eletrodo íonseletivo (ISELAB - Drake - São José do Rio Preto, SP, Brasil).

O método estatístico utilizado para avaliar as respostas foi a analise de variância com medidas repetidas. Comparações de médias aos pares foram feitas usando o Teste de TukeyKramer. Para todas as análises adotouse $\alpha=0,05$. As análises foram realizadas por meio do softwear SAS 9.1.3., SAS Institute Inc., USA.

\section{RESULTADOS}

A SPt não foi influenciada significativamente pelo fator tempo, e não houve diferença significativa entre as médias gerais dos grupos (Tabela 1). A comparação múltipla de médias indicou que não houve variação entre as médias ao longo do tempo (basal, 15, 30,45 e 60 dias) dentro de cada grupo (Tabela 2).

No perfil hepático a SAlb não foi influenciada significativamente pelos fatores tempo, doença ou tratamento, mas houve interação significativa entre tempo e doença, e não houve diferença significativa entre as médias gerais dos grupos (Tabela 1). A comparação múltipla de médias indicou que não houve variação entre as médias ao longo do tempo (basal, 15, 30, 45 e 60 dias) dentro de cada grupo (Tabela 2).
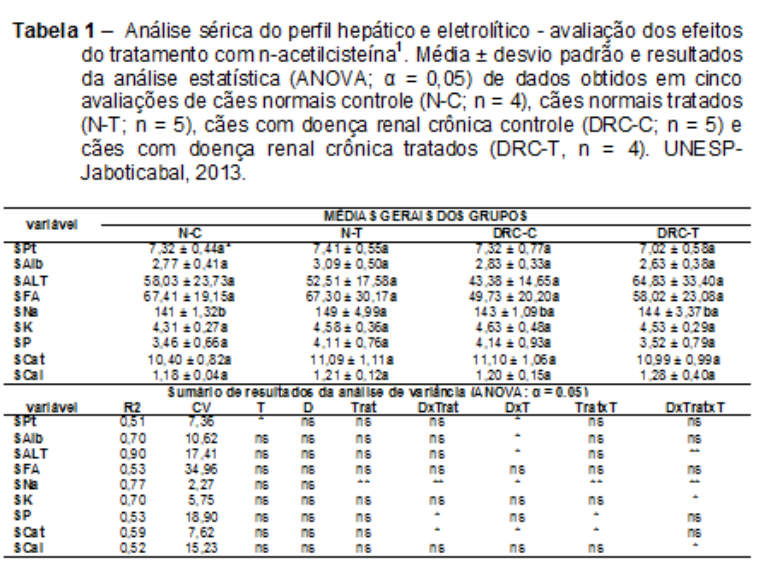
estatisticamente entre si (Tukey-Krame; $\alpha=0,05$ ). Variáveis - Sureia = ureia sérica (mg/dL): $S a d=$ creatinina sérica $(\mathrm{mg} / \mathrm{dL}): \mathrm{SPt}$ = proteina sérica (g/dL): SAlb = slbumina sérica (g/dL): SALT = slanina aminotransferase sérica (mg/dL): SFA fól

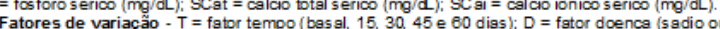
doente rensl crônico): Trat = fator tratamento ( $\mathbf{s e m}$ tratamento ou tratado com n-acefilcisteína)
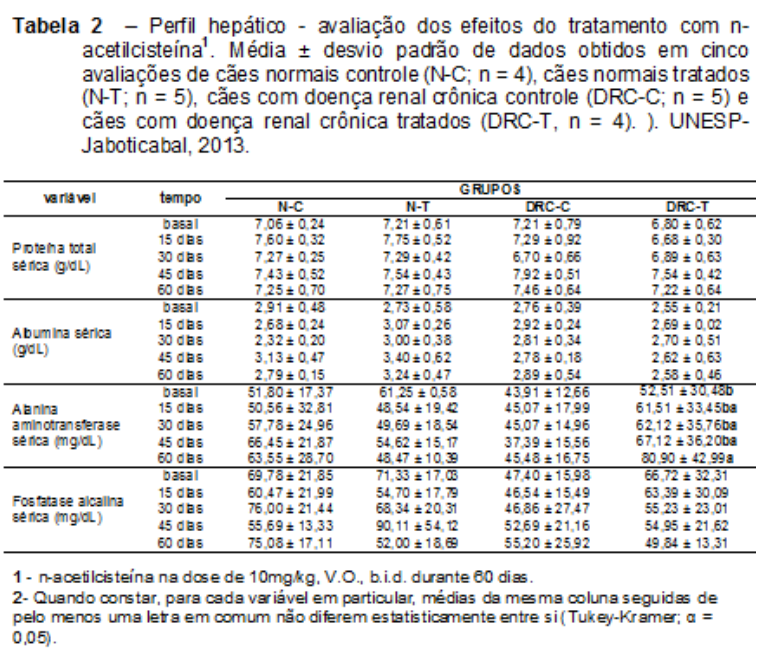

A SALT não foi influenciada significativamente pelos fatores tempo, doença ou tratamento, mas houve interação significativa entre tempo e doença, e não houve diferença significativa entre as médias gerais dos grupos (Tabela 1). A comparação múltipla de médias indicou que no grupo DRC-T as médias aumentaram gradativamente ao longo do tempo (basal, 15, 30, 45 e 60 dias) e foi verificada diferença significativa entre a média basal $(52,51 \mathrm{mg} / \mathrm{dL} \pm 30,48$ $\mathrm{mg} / \mathrm{dL})$ e a obtida aos 60 dias $(80,90$ $\mathrm{mg} / \mathrm{dL} \pm 42,99 \mathrm{mg} / \mathrm{dL}$ ), embora tenha havido variação grande entre os indivíduos do grupo (Tabela 2).

A SFA não foi influenciada significativamente pelos fatores analisados (tempo, doença e tratamento) e não houve diferença 
significativa entre as médias gerais dos grupos (Tabela 1). A comparação múltipla de médias indicou que não houve variação entre as médias ao longo do tempo (basal, 15, 30, 45 e 60 dias) dentro de cada grupo (Tabela 2).

A SNa foi influenciada significativamente pelo fator tratamento, mas todas as interações de fatores foram significativas. A comparação entre as médias gerais dos grupos mostrou que a média do grupo N-T (149 \pm $4,99 \mathrm{mEq} / \mathrm{L})$ foi significativamente maior do que a média do grupo N-C (141 \pm $1,32 \mathrm{mEq} / \mathrm{L})$, mas não diferiu das demais (Tabela 1). A comparação múltipla de médias, entretanto, não indicou variação significativa entre as médias ao longo do tempo (basal, 15, 30, 45 e 60 dias) dentro de cada grupo (Tabela 3 ).

O SK não foi influenciado significativamente pelos fatores tempo, doença ou tratamento, mas houve interação significativa entre doença, tratamento e tempo, e não houve diferença significativa entre as médias gerais dos grupos (Tabela 1). Mas, a comparação múltipla de médias indicou que no grupo N-T a média obtida aos 15 dias $(4,94 \pm 0,38 \mathrm{mEq} / \mathrm{L})$ foi significativamente maior do que a basal $(4,26 \pm 0,22 \mathrm{mEq} / \mathrm{L})$ e ambas não diferiram significativamente das demais média do grupo (Tabela 3).

O SP não foi influenciada significativamente pelos fatores tempo, doença ou tratamento, mas as interações entre doença e tratamento, e entre tratamento e tempo foram significativas. A comparação entre as médias gerais dos grupos mostrou que não houve diferença significativa (Tabela 1) e a comparação múltipla de médias em função do tempo também não indicou variação significativa entre as médias ao longo do tempo (basal, 15, 30,45 e 60 dias) dentro de cada grupo (Tabela 3).
Tabela 3 - Perfil eletrolítico - avaliação dos efeitos do tratamento com nacetilcisteína. Média \pm desvio padrão de dados obtidos em cinco avaliações de cães normais controle $(\mathrm{N}-\mathrm{C} ; \mathrm{n}=4)$, cães normais tratado $(\mathrm{N}-\mathrm{T} ; \hat{n}=5$ ), cães com doença renal đônica controle (DRC-C; $n=5$ ) cães com doença renal crônica tratados (DRC-T, $n=4)$.). UNESPJaboticabal, 2013.

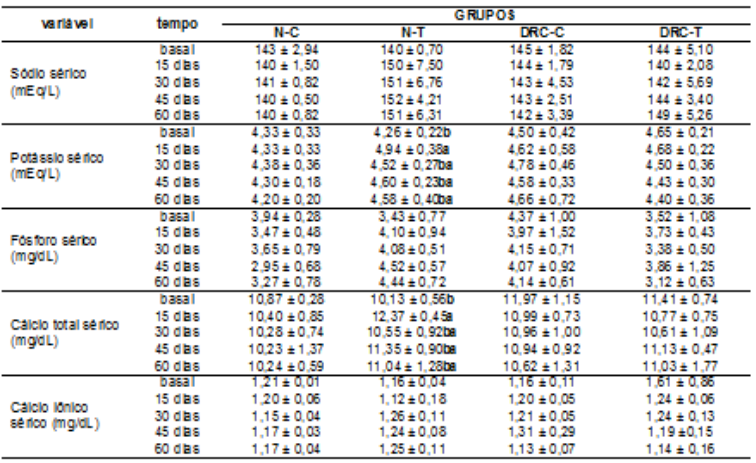

1- n-acefilcisteina na dose de $10 \mathrm{mg} / \mathrm{kg}$. V. O., b.i.d. durante 60 dias

O SCat não foi influenciada significativamente pelos fatores tempo, doença ou tratamento, mas houve interação significativa entre doença e tratamento, doença e tempo, e entre tratamento e tempo, e não houve diferença significativa entre as médias gerais dos grupos (Tabela 1). Mas, a comparação múltipla de médias indicou que no grupo N-T a média obtida aos 15 dias $(12,37 \pm 0,45 \mathrm{mg} / \mathrm{dL})$ foi significativamente maior do que a basal $(10,13 \pm 0,56 \mathrm{mg} / \mathrm{dL})$ e ambas não diferiram significativamente das demais média do grupo (Tabela 3).

A concentração de SCai não foi influenciada significativamente pelos fatores tempo, doença ou tratamento, mas houve interação significativa entre os três fatores. A comparação entre as médias gerais dos grupos mostrou que não houve diferença significativa (Tabela 1) e a comparação múltipla de médias em função do tempo também não indicou variação significativa entre as médias ao longo do tempo (basal, 15, 30,45 e 60 dias) dentro de cada grupo (Tabela 3).

\section{DISCUSSÃO}

Segundo Chew e Dibartola (1989), em relação às $\mathrm{SNa}$ e $\mathrm{SK}$, não são descritas alterações marcantes nos 
cães com DRC, já que os mecanismos de regulação do sódio e do potássio são preservados até os estágios avançados da enfermidade. No entanto, em um estudo realizado nos Laboratórios do Grupo de Pesquisa em Nefrologia e Urologia - UNESP - Jaboticabal, por Silva (2002), em cães com insuficiência renal crônica naturalmente adquirida, ficou demonstrado aumento significativo da excreção fracionada de sódio, e a diminuição significativa da concentração sérica deste íon. No nosso estudo foi constatado que o $\mathrm{SNa}$ não foi influenciado pelo fator doença, mas a média de sódio sérico do $\mathrm{N}$-T foi maior que a do $\mathrm{N}-\mathrm{C}$, indicando um possível efeito do antioxidante NAC encontrado somente nos cães normais. Com relação ao SK, embora as médias gerais dos grupos não tenham diferido significativamente entre si, os cães do $\mathrm{N}-\mathrm{T}$ tiveram aumento significativo da média aos 15 dias, sem redução significativa nos dias que se seguiram. Também no caso do $S K$, não se observou efeito do NAC no grupo DRC$\mathrm{T}$.

Newman (2012) descreveram que em pacientes com DRC ocorre aumento do SP e, consequentemente, estimulação da secreção de paratormônio caracterizando o quadro denominado hiperparatireoidismo secundário renal. Segundo estudo realizado por Nassar (2000), a presença de hiperfosfatemia foi constatada em $87 \%$ dos casos de cães com DRC e o aumento da concentração sérica de paratormônio em 93\%, observando-se correlação positiva entre os dois parâmetros. No nosso estudo não se observaram diferenças nas médias das concentrações SP, SCat ou SCai entre os grupos, evidenciando que os animais examinados ainda não se encontravam com déficit de excreção renal de fósforo ou de controle do cálcio. Entretanto, a exemplo do que ocorreu com outros eletrólitos, o SCat aumentou no grupo
$\mathrm{N}-\mathrm{T}$, com destaque significativo para a média obtida aos 15 dias. Neste caso especificamente, A média de concentração SCat obtida aos 15 dias chegou a ultrapassar o limite superior de normalidade, mas 15 dias depois já estava semelhante ao valor basal.

Segundo Center (1992) e Busch (2004) devido ao aumento da atividade osteoclástica associada nos doentes renais crônicos, podemos encontrar uma elevação SFA, que geralmente não excede de quatro a seis vezes ao seu valor máximo de normalidade. No presente trabalho não foi evidenciado diferença significativa das médias gerais entre os grupos $\mathrm{N}-\mathrm{C}, \mathrm{N}-\mathrm{T}$, DRC-C e DRC-T, e os valores obtidos, estavam dentro dos valores de normalidade. Como já mencionado, nossos pacientes não tinham sinais indicativos clínicos ou laboratoriais para dar suporte a um quadro de hiperparatireoidismo secundário renal e suas consequências sobre o fígado.

\section{CONCLUSÃO}

A utilização do antioxidante NAC promoveu em cães idosos sadios, o aumento das SK e SCat, além de aumentos não significativos da SAlb, não sendo observados efeitos colaterais nos animais estudados.

\section{AGRADECIMENTOS}

Ao Conselho Nacional de Desenvolvimento Científico e Tecnológico (CNPq) pela concessão do auxílio à pesquisa CNPq n. ${ }^{\circ} 474017 / 2008-7 ;$ ao Serviço de Nefrologia e Urologia Veterinária do Departamento de Clínica e Cirurgia Veterinária - Unesp- campus de Jaboticabal-SP pela concessão dos animais; a Faculdade de Ciências Agrárias e Veterinárias - Unesp campus de Jaboticabal-SP pela 
concessão dos laboratórios e de toda infraestrutura.

\section{NOTAS INFORMATIVAS}

A utilização de animais, seguindo o protocolo experimental do presente estudo foi previamente aprovada, pela Comissão de Ética no Uso de Animais da Faculdade de Ciências Agrárias e Veterinárias Unesp - campus de Jaboticabal-SP conforme processo n. ${ }^{\circ}$ 006823-09.

\section{REFERÊNCIAS}

BUSCH, B. M. Interpretação de resultados laboratoriais para clínicos de pequenos animais. São Paulo: Roca, 2004, 376p.

CENTER, S. A. Fisiopatologia e diagnóstico laboratorial das moléstias hepáticas. In: ETTINGER, S. J. Tratado de medicina veterinária interna. $3^{\circ}$ ed. São Paulo: Manole, 1992.Cap. 37, p. 123-132.

CHEW, D. J.; DIBARTOLA, S. P. Diagnosis and pathophysiology of renal disease. In:ETTINGER, S. J.; FELDMAN, E. C. Textbook of veterinary internal medicine, 3 ed. Philadelphia: W. B. Saunders, 1989. Cap. 257, p.1893-1961.

DIDONÉ, E. C.; CERSKI, C. T.; KALIL, N. Nacetilcisteína diminui a congestão hepática na lesão de isquemia e reperfusão - estudo experimental. Revista do Colégio Brasileiro de Cirurgia, v.29, n.04, p.191-196, 2002.

IRIS [2009]. International Renal Interest Society. Staging Chronic Kidney Disease (CKD) 2009. Disponível em: <http://www.iriskidney.com/pdf/IRIS\%20A4\%20Poster.pdf >. Acesso em 13/04/2012.

LOCATELLI, F.; CANAUD, B.; ECKARDT, K. U. et al. Oxidative stress in end-stage renal disease: an emerging threat to pacient outcome. Nephrology Dialysis Transplantation, Oxford, v.18, n.7, p.1272-1280, 2003.

NEWMAN, S. J. The urinary system. In: McGAVIN, M. D.; ZACHARY, J. F. Pathologic basis of veterinary disease. 5.ed. St. Louis: Elsevier, 2012, Cap. 11, p.589-657.

NASSAR, P. L. Avaliação da concentração sérica de paratormônio intacto (PTHi), pelo método imunofluorométrico, em cães hígidos e cães com insuficiência renal crônica em hiperazotemia. São Paulo 2000. 93f.

Dissertação (Mestrado em Medicina Veterinária)

- Curso de Pós-graduação em Medicina

Veterinária, Faculdade de Medicina Veterinária e Zootecnia, Universidade de São Paulo, São Paulo, 2000

PAPICH, M. G. Saunders Handbook of Veterinary Drugs. 2 th. St Louis: Elsevier, 2007, 774p.

SILVA, S.R.R. Pressão arterial e sua relação com a homeostase de sódio em cães com insuficiência renal crônica. 2002, 51Ff.

Dissertação de mestrado em medicina veterinaria - Programa de Pós-gradução em Medicina Veterinária, Faculdade de Ciências Agrárias e Veterinárias, Universidade Estadual Paulista, Jaboticabal 2002.

VATTIMO, M. F.; PINTO,C. F.; WATANABE, M. et al [2004]. Hiperhidratação ou NAC na prevenção da insuficiência renal aguda induzida por contraste iodado em ratos. In: XXII Congresso Brasileiro de Nefrologia. Salvador-BA, 18 a 22 de setembro de 2004. Disponível em: http://128.241.200.137/2632/v26e3s2p436p.pdf Acesso em: 10/04/2013.

ZENG, S.; LIN, Y.; DI, J. F. et al. Protective effect of $\mathrm{N}$-acetylcysteine on liver and lung in mice after ischemia-reperfusion injury. Xi Bao Yu Fen Zi Mian Yi Xue Za Zhi, v.11, n. 25, p.1058-1060, 2006. 